\title{
A NEW OSCILLATION CRITERION FOR TWO-DIMENSIONAL DYNAMIC SYSTEMS ON TIME SCALES
}

\author{
JIA BAOGUO
}

Abstract. Consider the linear dynamic system on time scales

$$
u^{\Delta}=p v, \quad v^{\Delta}=-q u^{\sigma}
$$

where $p>0$ and $q$ are rd-continuous functions on a time scale $\mathbb{T}$ such that $\sup \mathbb{T}=\infty$. When $q(t)$ is allowed to take on negative values, we establish an oscillation criterion for system (0.1). Our result improves a main result of Fu and Lin [S. C. Fu and M. L. Lin, Oscillation and nonoscillation criteria for linear dynamic systems on time scales, Computers and Mathematics with Applications, 59(2010), 2552-2565].

\section{Introduction}

Consider the linear dynamic system on time scales

$$
u^{\Delta}=p v, \quad v^{\Delta}=-q u^{\sigma}
$$

where $p(t)>0$ and $q(t)$ are rd-continuous functions on a time scale $\mathbb{T}$ such that $\sup \mathbb{\mathbb { N }}=\infty$. For convenience, we put $P(t)=\int_{t_{0}}^{t} p(s) \Delta s$. A solution $(x(t), y(t))$ of system (1.1) is called oscillatory if both $x(t)$ and $y(t)$ are oscillatory functions, and otherwise it will be called nonoscillatory. System (1.1) will be called oscillatory if its solutions are oscillatory. System (1) can be reduced to a single dynamic equation

$$
\left(\frac{1}{p} u^{\Delta}\right)^{\Delta}+q u^{\sigma}=0
$$

In [1], the following oscillation theorem is obtained.

Theorem 1.1. Suppose that $p(t)$ and $q(t)$ are nonnegative and $\int_{t_{0}}^{\infty} p(s) \Delta s=\infty, \lim _{t \rightarrow \infty} \frac{\mu(t) p(t)}{P(t)}=$ 0 . Suppose also that there exists $\lambda \in(0,1)$ such that

$$
\int_{t_{0}}^{\infty} P^{\lambda}(t) q(t) \Delta t=\infty
$$

then system (1.1) is oscillatory.

Corresponding author: .

2000 Mathematics Subject Classification. 34K11, 39A10, 39A99.

Key words and phrases. Two-dimensional, oscillation, time scale, dynamic equation.

*This work is supported by the National Natural Science Foundation of China (No.10971232). 
In this paper, we prove the above Theorem 1.1 when $q(t)$ is allowed to take on negative values. As applications, we get that the linear differential system

$$
x^{\prime}=t^{a-1} y, \quad y^{\prime}=-\left(\frac{b}{t^{\frac{a}{2}+1}}+\frac{\sin c t}{t^{\frac{a}{2}}}\right) x
$$

and the linear difference system

$$
\Delta x(n)=n y(n), \quad \Delta y(n)=-\left(\frac{b}{n^{2}}+\frac{(-1)^{n}}{n}\right) x(n+1)
$$

where $a>0, b>0, c \in R$, are oscillatory.

\section{Some lemmas}

Lemma 2.1. If $(u(t), v(t))$ is a nonoscillatory solution of system (1.1), then the component $u(t)$ is also nonoscillatory.

Proof. Assume that $(u(t), v(t))$ is a solution of system (1.1) and $u(t)$ is oscillatory, but $v(t)$ is nonoscillatory. Without loss of generality, we let $v(t)>0$ on $\left[t_{0}, \infty\right)_{\mathbb{V}}$. In view of the first equation of system (1.1), we have $u^{\Delta}(t)>0$ on $\left[t_{0}, \infty\right)_{\mathbb{T}}$. Thus, $u(t)>0$ or $u(t)<0$ for all large $t$, which leads to a contradiction. So, the oscillation of $u(t)$ implies that of $v(t)$ as well.

The following two lemmas may also be found in [3].

Lemma 2.2. Assume $a \in \mathbb{T}$, let $\omega=\sup \mathbb{T}$. If $\omega<\infty$, then we assume $\rho(\omega)=\omega$. If equation (1.2) is nonoscillatory on $[a, \omega)$, then there is a solution $u_{1}$, called a recessive solution at $\omega$, such that for any second linearly independent solution $u_{2}$, called a dominant solution at $\omega$, we have

$$
\lim _{t \rightarrow \omega^{-}} \frac{u_{1}(t)}{u_{2}(t)}=0, \int_{b}^{\omega} \frac{p(t)}{u_{1}(t) u_{1}^{\sigma}(t)} \Delta t=\infty \text {, and } \int_{b}^{\omega} \frac{p(t)}{u_{2}(t) u_{2}^{\sigma}(t)} \Delta t<\infty,
$$

where $b<\omega$ is sufficiently close. Furthermore,

$$
\frac{u_{2}^{\Delta}(t)}{p(t) u_{2}(t)}>\frac{u_{1}^{\Delta}(t)}{p(t) u_{1}(t)}
$$

for $t<\omega$ sufficientlyclose.

Lemma 2.3. (Picone's Identity). Assume $u(t)$ is a positive solution of $(1.2), z(t)=\frac{u^{\Delta}(t)}{p(t) u(t)}$ on $[a, \infty)$ and assume $h: \mathbb{T} \rightarrow \mathbb{R}$ is a continuously differentiable function. Then we have for all $t \in[a, \infty)$,

$$
\begin{aligned}
{\left[z h^{2}\right]^{\Delta}(t)=} & -q(t) h^{2}(\sigma(t))+\frac{\left[h^{\Delta}(t)\right]^{2}}{p(t)} \\
& -\left[\frac{z(t) h^{\sigma}(t)}{\sqrt{\frac{1}{p(t)}+\mu(t) z(t)}}-\sqrt{\frac{1}{p(t)}+\mu(t) z(t)} h^{\Delta}(t)\right]^{2} .
\end{aligned}
$$


The following theorem may be found in [6], Theorem 11, Page 17-18.

Theorem 2.4. The equation $\left(\frac{1}{p(t)} u^{\prime}(t)\right)^{\prime}+q(t) u(t)=0$ is oscillatory on the interval $\left[t_{0}, \infty\right)$, if $\int_{t_{0}}^{\infty} p(t) d t=\infty$ and there exists a continuously differentiable function $h(t)>0$ such that

$$
\int_{t_{0}}^{\infty}\left[q(t) h^{2}(t)-\frac{1}{p(t)}\left(h^{\prime}(t)\right)^{2}\right] d t=+\infty
$$

Analogous to the above theorem, we may obtain a corresponding time scales version which we state as follows:

Lemma 2.5. If $\int_{t_{0}}^{\infty} p(t) \Delta t=\infty$ and there exists a function $h \in C_{r d}^{1}\left[t_{0}, \infty\right)$ such that $h(t)>0$ on $\left[t_{0}, \infty\right)$ and

$$
\int_{t_{0}}^{\infty}\left[q(t) h^{2}(\sigma(t))-\frac{1}{p(t)}\left[h^{\Delta}(t)\right]^{2}\right] \Delta t=+\infty .
$$

Then the dynamic equation (1.2) is oscillatory on $\left[t_{0}, \infty\right) \mathbb{\pi}$.

Proof. Assume that (1.2) is nonoscillatory. By Lemma 2.2, there is a dominant solution $u_{2}(t)>$ 0 at $\infty$ such that for $t_{1}>t_{0}$, sufficiently large,

$$
\int_{t_{1}}^{\infty} \frac{p(t)}{u_{2}(t) u_{2}^{\sigma}(t)} \Delta t<\infty
$$

Let $z(t)=\frac{u_{2}^{\Delta}(t)}{p(t) u_{2}(t)}$. We have

$$
z^{\Delta}(t)=-q(t)-\frac{z^{2}(t)}{\frac{1}{p(t)}+\mu(t) z(t)},
$$

and

$$
\frac{1}{p(t)}+\mu(t) z(t)=\frac{u_{2}(\sigma(t))}{p(t) u_{2}(t)}>0
$$

From lemma 2.3, we get

$$
\begin{aligned}
{\left[z h^{2}\right]^{\Delta}(t) } & =-q(t) h^{2}(\sigma(t))+\frac{\left[h^{\Delta}(t)\right]^{2}}{p(t)} \\
& -\left[\frac{z(t) h^{\sigma}(t)}{\sqrt{\frac{1}{p(t)}+\mu(t) z(t)}}-\sqrt{\frac{1}{p(t)}+\mu(t) z(t)} h^{\Delta}(t)\right]^{2} \\
& \leq-q(t) h^{2}(\sigma(t))+\frac{\left[h^{\Delta}(t)\right]^{2}}{p(t)} .
\end{aligned}
$$

Integrating from $t_{1}$ to $t$, we get

$$
z(t) h^{2}(t) \leq z\left(t_{1}\right) h^{2}\left(t_{1}\right)-\int_{t_{1}}^{t}\left[q(t) h^{2}(\sigma(t))-\frac{\left[h^{\Delta}(t)\right]^{2}}{p(t)}\right] \Delta t
$$


By (2.3), we obtain

$$
\lim _{t \rightarrow \infty} z(t) h^{2}(t)=-\infty
$$

So there exists a large $t_{2}>t_{1}$ such that for $t>t_{2}$, we have

$$
z(t)=\frac{u_{2}^{\Delta}(t)}{p(t) u_{2}(t)}<0
$$

This implies $u_{2}^{\Delta}(t)<0$ for $t>t_{2}$. Hence

$$
\begin{aligned}
\int_{t_{2}}^{\infty} p(t) \Delta t & =u_{2}\left(t_{2}\right) u\left(\sigma\left(t_{2}\right)\right) \int_{t_{2}}^{\infty} \frac{p(t)}{u_{2}\left(t_{2}\right) u_{2}\left(\sigma\left(t_{2}\right)\right)} \Delta t \\
& \leq u_{2}\left(t_{2}\right) u\left(\sigma\left(t_{2}\right)\right) \int_{t_{2}}^{\infty} \frac{p(t)}{u_{2}(t) u_{2}(\sigma(t))} \Delta t \\
& <\infty
\end{aligned}
$$

which is a contradiction.

From Lemma 2.1 and Lemma 2.5, we can get the following

Lemma 2.6. Under the assumption of Lemma 2.5, the system (1.1) is oscillatory.

Let $\hat{\mathbb{T}}:=\{t \in \mathbb{T}: \mu(t)>0\}$ and let $\chi$ denote the characteristic function of $\hat{\mathbb{T}}$. The following condition, which will be needed later, imposes a lower bound on the graininess function $\mu(t)$, for $t \in \hat{\mathbb{T}}$. More precisely, we introduce the following (see [4] and [5]).

Condition (C). We say that $\mathbb{T}$ satisfies condition $C$ if there is an $M>0$ such that

$$
\chi(t) \leq M \mu(t), \quad t \in \mathbb{T} .
$$

We note that if $\mathbb{T}$ satisfies condition (C), then the set

$$
\check{\mathbb{T}}=\{t \in \mathbb{T} \mid t>0 \text { is isolated or right-scattered or left-scattered }\}
$$

is necessarily countable.

Lemma 2.7. Assume that $\mathbb{1}$ satisfies condition $C$. Then for all $\lambda \in[0,1)$, we have

$$
\int_{t_{0}}^{t} \frac{p(s)}{P^{2-\lambda}(\sigma(s))} \Delta s \leq \frac{\left[P\left(t_{0}\right)\right]^{-1+\lambda}}{1-\lambda} .
$$

Proof. For any $t \in \mathbb{T}$, if $t=t^{\prime}<t^{\prime \prime}=\sigma(t)$, from $P^{\Delta}(s)=p(s)>0$, we get that

$$
\int_{t}^{\sigma(t)} \frac{p(s)}{P^{2-\lambda}(\sigma(s))} \Delta s=\frac{\mu(t) P^{\Delta}(t)}{P^{2-\lambda}(\sigma(t))}
$$




$$
\begin{aligned}
& =\frac{P(\sigma(t))-P(t)}{P^{2-\lambda}(\sigma(t))} \\
& \leq \int_{P(t)}^{P(\sigma(t))} \frac{1}{v^{2-\lambda}} d \nu .
\end{aligned}
$$

If the real interval $\left[t^{\prime}, t^{\prime \prime}\right] \subset \mathbb{T}$, then

$$
\int_{t^{\prime}}^{t^{\prime \prime}} \frac{p(s)}{P^{2-\lambda}(\sigma(s))} \Delta s=\int_{t^{\prime}}^{t^{\prime \prime}} \frac{p(s)}{P^{2-\lambda}(s)} d s=\int_{P\left(t^{\prime}\right)}^{P\left(t^{\prime \prime}\right)} \frac{1}{v^{2-\lambda}} d v .
$$

Note that since $\mathbb{I}$ satisfies condition $C$, we have from (2.5), (2.6) and the additivity of the integral that

$$
\begin{aligned}
\int_{t_{0}}^{t} \frac{p(s)}{P^{2-\lambda}(\sigma(s))} \Delta s & \leq \int_{P\left(t_{0}\right)}^{P(t)} \frac{1}{v^{2-\lambda}} d v \\
& =\frac{[P(t)]^{-1+\lambda}-\left[P\left(t_{0}\right)\right]^{-1+\lambda}}{-1+\lambda} \\
& \leq \frac{\left[P\left(t_{0}\right)\right]^{-1+\lambda}}{1-\lambda} .
\end{aligned}
$$

\section{Main Theorem}

Theorem 3.1. Assume that $\mathbb{T}$ satisfies condition $C$ and $\lim _{t \rightarrow \infty} \frac{\mu(t) p(t)}{P(t)}=0, \int_{t_{0}}^{\infty} p(t) \Delta t=\infty$. Suppose also that there exists $\lambda \in[0,1)$ such that

$$
\int_{t_{0}}^{\infty} P^{\lambda}(\sigma(t)) q(t) \Delta t=\infty
$$

Then system (0.1) is oscillatory.

Proof. Take $h(t)=[P(t)]^{\frac{\lambda}{2}}$. Using the Pötzsche chain rule [3, Theorem 1.90], we get

$$
h^{\Delta}(t)=\frac{\lambda}{2} \int_{0}^{1}[(1-\tau) P(t)+\tau P(\sigma(t))]^{\frac{\lambda}{2}-1} d \tau p(t) .
$$

Since $\lim _{t \rightarrow \infty} \frac{\mu(t) p(t)}{P(t)}=0$, it follows that $\lim _{t \rightarrow \infty} \frac{P(\sigma(t))}{P(t)}=1$ so given $\epsilon$ with $0<\epsilon<1$, there exists $t_{1}$ sufficiently large so that for $t \geq t_{1}$ we have

$$
P(t) \geq(1-\epsilon) P(\sigma(t))
$$

From (3.2), (3.3) and Lemma 2.7, we get

$$
\begin{aligned}
\int_{t_{1}}^{\infty} \frac{\left[h^{\Delta}(t)\right]^{2}}{p(t)} \Delta t & \leq\left(\frac{\lambda}{2}\right)^{2} \int_{t_{1}}^{\infty} p(t)[P(\sigma(t))]^{\lambda-2}\left\{\int_{0}^{1}[(1-\tau)(1-\epsilon)+\tau]^{\frac{\lambda}{2}-1} d \tau\right\}^{2} \Delta t \\
& =\frac{1}{\epsilon^{2}}\left[1-(1-\epsilon)^{\frac{\lambda}{2}}\right]^{2} \int_{t_{1}}^{\infty} p(t)[P(\sigma(t))]^{\lambda-2} \Delta t
\end{aligned}
$$




$$
<+\infty
$$

From (3.1) and (3.4), we obtain

$$
\int_{t_{1}}^{\infty}\left[q(t) h^{2}(\sigma(t))-\frac{1}{p(t)}\left[h^{\Delta}(t)\right]^{2}\right] \Delta t=+\infty
$$

From (3.5) and Lemma 2.6, the system (1.1) is oscillatory.

Remark 3.2. From the proof of the Theorem 3.1, it is easy to see that the assumption " $\lim _{t \rightarrow \infty}$ $\frac{\mu(t) p(t)}{P(t)}=0$ " in Theorem 3.1 can be replaced by " $\frac{P(\sigma(t))}{P(t)}$ is bounded for all $t \in \mathbb{T}$ ".

Let $\mathbb{T}=\mathbb{N}$. Consider the linear difference system

$$
\Delta x(n)=2^{n} y(n), \quad \Delta y(n)=-\frac{\alpha+\beta(-1)^{n}}{\left[2^{n+1}-1\right]^{\frac{1}{2}}} x(n+1)
$$

where $\beta>\alpha>0$. It is easy to see that $P(t)=\int_{0}^{t} p(t) \Delta t=2^{t}-1 . \lim _{t \rightarrow \infty} \frac{p(t)}{P(t)}=1 \neq 0 . \frac{P(\sigma(t))}{P(t)}=$ $\frac{2^{t+1}-1}{2^{t}-1}$ is bounded for $t \geq 1$. Choose $\lambda=1 / 2$. We have

$$
\int_{0}^{\infty} P^{\frac{1}{2}}(\sigma(t)) q(t) \Delta t=\sum_{n=1}^{\infty}\left(\alpha+\beta(-1)^{n}\right)=\infty .
$$

So by Remark 3.2, (3.6) is oscillatory, but do not follow from Theorem 3.1.

\section{Example}

Example 4.1. Consider the linear differential system

$$
x^{\prime}=t^{a-1} y, \quad y^{\prime}=-\left(\frac{b}{t^{\frac{a}{2}+1}}+\frac{\sin c t}{t^{\frac{a}{2}}}\right) x
$$

where $a>0, b>0, c \in R$.

Take $\lambda=\frac{1}{2}$. It is easy to see that

$$
\begin{gathered}
P(t)=\int_{1}^{t} p(s) d s=\frac{1}{a}\left(t^{a}-1\right), \\
\int_{1}^{\infty} P^{\frac{1}{2}}(t) q(t) d t=\frac{b}{\sqrt{a}} \int_{1}^{\infty}\left[\frac{t^{a}-1}{t^{a+2}}\right]^{\frac{1}{2}} d t+\frac{1}{\sqrt{a}} \int_{1}^{\infty}\left(1-t^{-a}\right)^{\frac{1}{2}} \sin c t d t .
\end{gathered}
$$

By using Taylor's expansion, there exists a positive integer $m$ such that $m a>1$ and

$$
\begin{aligned}
\left(1-t^{-a}\right)^{\frac{1}{2}} & =1-2^{-1} t^{-a}+\frac{2^{-1}\left(2^{-1}-1\right)}{2 !} t^{-2 a}+\cdots \\
& +(-1)^{m} \frac{2^{-1}\left(2^{-1}-1\right) \cdots\left(2^{-1}-m+1\right)}{m !} t^{-m a}+o\left(t^{-m a}\right) .
\end{aligned}
$$

Note that for $k=1,2, \cdots, m-1, \int_{1}^{\infty} t^{-k a} \sin c t d t$ and $\int_{1}^{\infty} o\left(t^{-m a}\right) d t$ are convergent. It is obvious that $\int_{1}^{\infty}\left[\frac{t^{a}-1}{t^{a+2}}\right]^{\frac{1}{2}} d t=+\infty$. So we have $\int_{1}^{\infty} P^{\frac{1}{2}}(t) q(t) d t=+\infty$. By Theorem 3.1, the system (4.1) is oscillatory. 
Example 4.2. Consider the linear difference system

$$
\Delta x(n)=n y(n), \quad \Delta y(n)=-\left(\frac{b}{n^{2}}+\frac{(-1)^{n}}{n}\right) x(n+1)
$$

where $b>0$.

Take $\lambda=\frac{1}{2}$. It is easy to see that $P(n)=\frac{n(n-1)}{2}$,

$$
\int_{1}^{\infty} P^{\frac{1}{2}}(t) q(t) \Delta t=\frac{1}{\sqrt{2}} \sum_{n=1}^{\infty}[n(n-1)]^{\frac{1}{2}}\left(\frac{b}{n^{2}}+\frac{(-1)^{n}}{n}\right) .
$$

Using Taylor's formula, we have

$$
\left(1-\frac{1}{n}\right)^{\frac{1}{2}}=1-\frac{1}{2 n}+\frac{2^{-1}\left(2^{-1}-1\right)}{2} \frac{1}{n^{2}}+o\left(n^{-2}\right) .
$$

So for any $m, \sum_{n=1}^{m}(-1)^{n}\left(1-\frac{1}{n}\right)^{\frac{1}{2}}$ is bounded. Therefore $\int_{1}^{\infty} P^{\frac{1}{2}}(t) q(t) d t=+\infty$. By Theorem 3.1 , the system (4.2) is oscillatory.

Example 4.3. Consider the $q$-difference system

$$
x^{\Delta}(t)=t^{a-1} y(t), \quad y^{\Delta}(t)=-\left(\frac{b+(-1)^{n}}{t^{\frac{a}{2}}}\right) x(q t) .
$$

where $a>0,0<b<1, t=q^{n} \in \mathbb{T}=q^{\mathbb{N}_{0}}, q>1$.

Take $\lambda=\frac{1}{2}$. It is easy to see that $P(t)=\frac{(q-1)\left(t^{a}-1\right)}{q^{a}-1}, P(\sigma(t))=\frac{(q-1)\left[(q t)^{a}-1\right]}{q^{a}-1}$. So $\lim _{t \rightarrow \infty} \frac{P(\sigma(t))}{P(t)}$ $=q^{a}$, which implies $\frac{P(\sigma(t))}{P(t)}$ is bounded.

$$
\int_{1}^{\infty} P^{\frac{1}{2}}(t) q(t) \Delta t=\left(\frac{q-1}{q^{a}-1}\right)^{\frac{1}{2}} \sum_{i=1}^{\infty}\left(\frac{q^{(i-1) a}-1}{q^{(i-1) a}}\right)^{\frac{1}{2}}\left[b+(-1)^{i}\right]=+\infty .
$$

By Theorem 3.1 and Remark 3.2, the system (4.3) is oscillatory.

\section{Acknowledgement}

The author is very grateful to referee for his/her valuable suggestions.

\section{References}

[1] Shengchen Fu, Mingli Lin, Oscillation and nonoscillation criteria for linear dynamic equations on time scales, Computers and Mathematics with Applications, 59(2010), 2552-2565.

[2] Youjun $\mathrm{Xu}$, Zhiting Xu, Oscillation criteria for two-dimensional dynamic systems on time scales, J. Computational and Applied Mathematics, 225(2009), 9-19.

[3] M. Bohner and A. Peterson, Dynamic Equation on Time Scales: An Introduction with Applications, Birkhäuser, Boston, 2001. 
[4] Lynn Erbe, Oscillation criteria for second order linear equations on a time scale, Canad. Appl. Math. Quart., 9(2001), 346-375.

[5] Jia Baoguo, Lynn Erbe and Allan Peterson, A Wong-type oscillation theorem for second order linear dynamic equations on time scales, J. Difference Equs. Appl., 16(2010), 15-36.

[6] W. A. Coppel, Disconjugacy, Lecture Notes In Mathematics, Springer-Verlag, No. 220, 1971, 17-18.

Department of Mathematics, Zhongshan University, Guangzhou, China 510275.

E-mail: mcsjbg@mail.sysu.edu.cn 